\title{
ABORDAGEM DA LATENCIAÇÃO DE FÁRMACOS COMO FERRAMENTA PARA DESCOBERTA DE NOVOS ANTICHAGÁSICOS
}

SERAFIM, Eliana Ometto Pavan. Departamento de Química Orgânica - Instituto de Química - Unesp. CP 355 - 14801-970. Araraquara-SP.

CHIN, Chung Man. Departamento de Fármacos e Medicamentos - Faculdade de Ciências Farmacêuticas Unesp. CP 502 - 14801-902. Araraquara-SP.

RIBEIRO, Maria Lúcia. Docente-pesquisador do Programa de Mestrado em Desenvolvimento Regional e Meio Ambiente do Centro Universitário de Araraquara - Uniara. Rua Carlos Gomes, 1338 - Centro.

CEP 14801-340. Araraquara-SP.

ARAÚJO, Danielle dos Santos. Aluna de IC do Programa de Mestrado em Desenvolvimento Regional e Meio Ambiente do Centro Universitário de Araraquara - Uniara. Bolsista pela Fundação Nacional de Desenvolvimento do Ensino Superior Particular - Funadesp.

\section{RESUMO}

A doença de Chagas, descoberta por Carlos Chagas há mais de um século, ainda constitui um grave problema de Saúde Pública. É considerada doença extremamente negligenciada, uma vez que afeta particularmente as populações dos países em desenvolvimento. Os pacientes dessa doença são, em sua maioria, de baixa renda e, não representando mercado, ficam excluídos do escopo e dos esforços de Pesquisa e Desenvolvimento das Indústrias Farmacêuticas. Estima-se que entre 8 e 11 milhões de indivíduos estejam infectados pelo Trypanosoma cruzi, agente etiológico da doença, e em, aproximadamente, 100 milhões, a população em risco na América Latina. Considerando que o tratamento da doença de Chagas continua sendo um desafio, pois apenas dois fármacos nitro-heterocíclicos são comercializados no mundo: nifurtimox e benznidazol, sendo este último o único fármaco disponível no mercado brasileiro; no entanto, esses fármacos são ativos na fase aguda da doença e o tratamento é ineficaz em pacientes na fase crônica, portanto, é relevante o desenvolvimento de agentes antichagásicos eficazes, principalmente para a fase crônica da doença. Este trabalho aborda a importância da latenciação para o desenvolvimento de novos pró-fármacos. Diversas técnicas metodológicas vêm sendo descritas na literatura, permitindo avanços significativos no planejamento e desenvolvimento de novos agentes antichagásicos, com ênfase na busca de pró-fármacos que permitemo aprimoramento do fármaco matriz.

Palavras-chave: Doença de Chagas; Agentes antichagásicos; Pró-fármacos.

\section{Abstract}

Chagas disease, discovered more than one century ago by Carlos Chagas, is still a serious Public Health problem. It is considered an extremely neglected disease, for it affects specially the population of developing countries. The patients with this disease have, in the great majority, low income and, for not representing market, they are excluded from the aims and efforts of research and development of pharmaceutical industries. About 8 to 11 million people may be infected with Trypanosoma cruzi, the etiological agent of the disease and about 100 million people are in risk in Latin America. The treatment of the disease is still a challenge, for only two nytroheterociclic drugs are commercialized in the world: nifurtimox and benznidazole, being this last one, the only available drug in the Brazilian market. However, these drugs are active only in the acute phase of the disease, and the treatment is not efficient in patients in the chronic phase. Consequently it is relevant to develop efficient anti-chagas compounds, particularly for the chronic phase of the disease. This paper discusses the importance 
of latency for the development of new pro-drugs. The literature describes several methodological techniques that have enabled significant advances in the planning and development of new anti-chagas agents, with emphasis on the search for pro-drugs that allow the enhancement of drug matrices.

KeYwords: Chagas disease; Anti-chagas compounds; Pro-drugs.

\section{Doença de Chagas}

Cerca de 3,5 bilhões de indivíduos no mundo sofrem de algum tipo de infecções parasitárias causadas por protozoários ou helmintos patogênicos (TRACY et al., 2001; PRATA\& DIAS \& COURA, 2011). Em países subdesenvolvidos, a incidência das doenças parasitárias é favorecida. Essas infecções estão relacionadas com as baixas condições socioeconômicas somadas à falta de educação e saúde (DIAS et al., 2009). Como consequência, ocorre o comprometimento do estado físico do hospedeiro, diminuindo sua resistência (TRACY et al., 2001; MARTINEZ et al., 2005). Outros fatores incluem a alta densidade populacional, controle inadequado dos vetores e reservatórios responsáveis por essas infecções, aumento da migração populacional, operações militares, principalmente, a falta de fármacos eficazes utilizados na quimioterapia (TRACY et al., 2001, DRUMOND \& MARCOPITO, 2006; IRIBARNE et al., 2007).

Adoença de Chagas ou Tripanossomíase Americana é causada pelo protozoário flagelado Trypanosoma cruzi. A etapa mais importante de transmissão do $T$. cruzi é efetuada por insetos da família Reduviidae, incluindo as espécies Panstrongylus megistus, Triatoma infestans e Rhodnius prolixus. No Brasil, o transmissor $T$. infestans é conhecido vulgarmente como "barbeiro", devido ao seu hábito de picar a face das pessoas (URBINA et al., 2003; WILLIAMSBLANGERO et al., 2003; WHO, 2011).

Atualmente, de 8 a 11 milhões de pessoas estão infectadas pelo $T$. cruzi e cerca de 5 a 6 milhões delas têm desenvolvido complicações crônicas incuráveis, tais como lesões cardíacas, neurológicas e desordens digestivas. A abrangência dessa doença ocorre desde o Canadá, Estados Unidos até a Argentina e Chile. No total, 22 países são endêmicos, principalmente nas Américas Central e do Sul. Dados da Iniciativa de Medicamentos para Doenças Negligenciadas indicam 14.000 mortes/ano e cerca de 100 milhões de indivíduos em risco de infecção na América Latina (AGUIRRE et al., 2005; RIGOL et al., 2005; COURA \& VINÃS, 2010; DNDi, 2011). A doença de Chagas, juntamente com outras parasitoses (malária, leishmaniose e esquistossomose), é uma das doenças parasitárias mais graves da América Latina, com alto impacto socioeconômico (NWAKA \& HUDSON, 2006).

No Brasil, a doença espalha-se por uma área de 3.600.000 de quilômetros quadrados, envolvendo 2.493 municípios e deixando sob risco de infecção 28 milhões de pessoas. A população infectada chega a 2,5 milhões de indivíduos infectados por T. cruzi, sendo $60 \%$ na área urbana (AMATO-NETO \& PASTERNAK, 2009), e compreendem, em sua maioria, indivíduos de origem rural e pertencentes aos níveis socioeconômicos mais baixos, contaminados através de fezes infectadas de triatomíneos. É estimado que de 25 a $30 \%$ dos indivíduos infectados terão seus quadros patológicos evoluídos para danos irreversíveis (TDR, 2005).

A morbimortalidade da Doença de Chagas no Brasil é significativa, vitimando $25 \%$ ou mais dos indivíduos infectados, que adquirem cardiopatia chagásica crônica evolutiva (DIAS, 2000; SDR, 2002; SZAJNMAN et al., 2005).

\section{Ciclo evolutivo e vias de transmissão}

O ciclo evolutivo do $T$. cruzi inicia-se pela ingestão, pelo vetor triatomíneo, de sangue infectado contendo as formas tripomastigotas do parasito.

Os parasitas ingeridos sofrem diferenciação para a forma epimastigota no lúmen intestinal do inseto, local onde ocorre a proliferação parasitária. Os parasitas, ao atingir a parte posterior do intestino do inseto vetor, modificam-se para formas tripomastigotas metacíclicas, que são as formas infectantes do parasita. Estas são 
eliminadas pelas fezes e urina do triatomíneo.

Dentro do organismo do vertebrado, as formas tripomastigotas metacíclicas do parasita passam por transformação para as formas amastigotas, reproduzindose por fissão binária. Dentro do hospedeiro as formas amastigotas se transformam em tripomastigotas, que são liberadas nos vasos periféricos e estarão aptos para infectar novas células em outros pontos do organismo, como músculos lisos e tecido nervoso. Assim, a transformação de amastigotas para tripomastigotas pode ocorrer emoutros pontos do organismo, continuando o ciclo da infecção (BRENER \& CANÇADO, 1979; DIAS, 1990; SZAJNMAN et al., 2005).

\section{COMPOSTOS NITRO-HETEROCí́CLICOS COM ATIVIDADE} na doença de Chagas

O grupo de compostos nitro-heterocíclicos biologicamente ativos contra o T. cruzi inclui vários 5e 2-nitroimidazóis e 5-nitrofuranos (Figura 1), os quais podem ser utilizados como agentes terapêuticos contra uma grande variedade de protozoários e bactérias anaeróbias, patogênicas em humanos e animais. Dentre esses compostos estão: benznidazol (I), nifurtimox (II), furazolidona (III), metronidazol (IV), secnidazol (V), ornidazol (VI) e tinidazol (VII), todos com amplo espectro de ação (RAETHER \& HÃNEL, 2003; DIAS, et al., 2009).

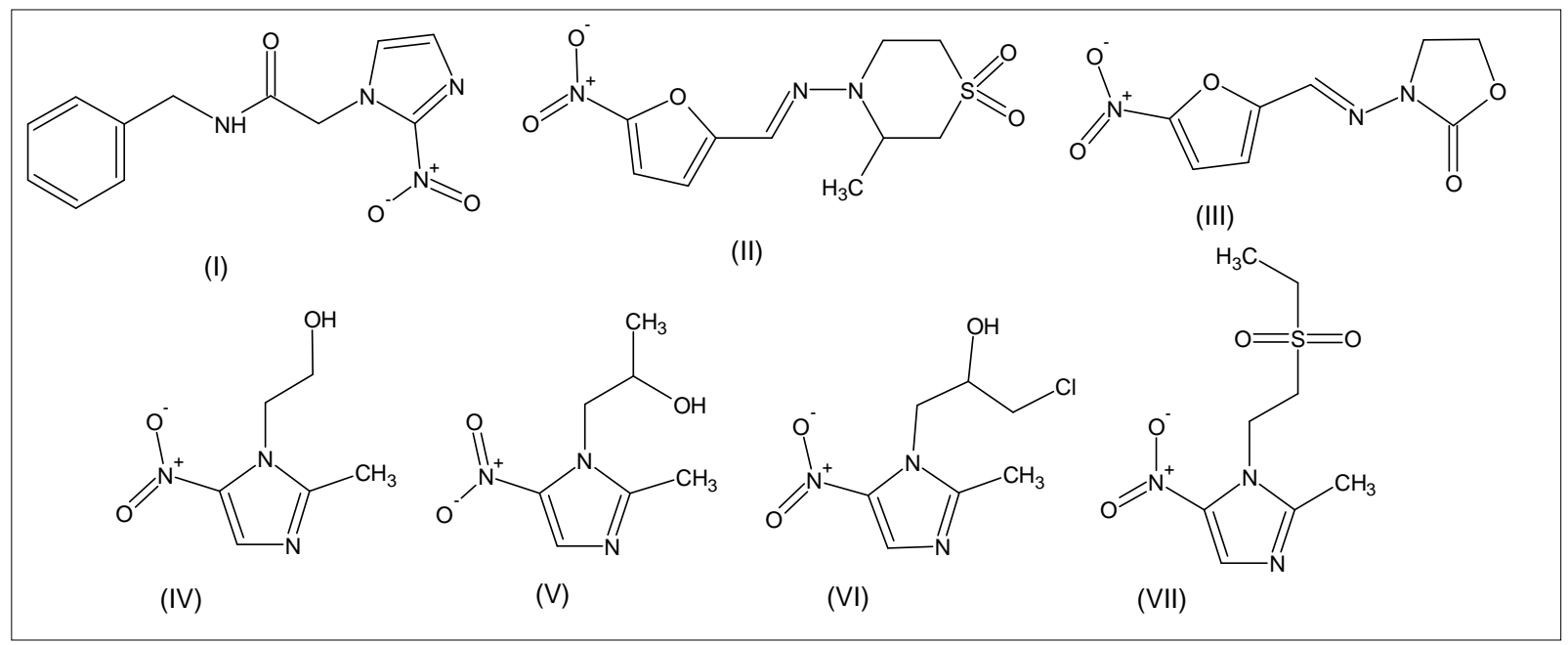

Figura 1 - Estrutura química dos compostos benznidazol (I), nifurtimox (II), furazolidona (III), metronidazol (IV), secnidazol(V), ornidazol (VI) e tinidazol (VII) utilizados como antiprotozoários e antimicrobianos.

Desde a década de 1970 até os dias de hoje, apenas dois fármacos estão disponíveis no mercado mundial para o tratamento da doença de Chagas; o nifurtimox (Lampit $^{\circledR}$, Bayer) e o benznidazol(Radanil ${ }^{\circledR}$, Rochagan $^{\circledR}$, Roche) (ANANDAN, 1997; TRACY et al., 2001; COURA \& CASTRO, 2002; OTERO et al., 2006; DIAS, et al., 2009). No entanto, esses fármacos são ativos apenas na fase aguda da doença e o tratamento é ineficaz em pacientes na fase crônica (URBINA etal., 2003; SDR, 2002; BOIANI et al., 2006). No Brasil, apenas o benznidazol está disponível no mercado.

Associando a grande incidência de parasitoses em nosso país ao fato de as cepas brasileiras de Trypanosoma cruzi serem resistentes ao nifurtimox, e à ameaça de suspensão da produção do benznidazol pela Roche no Brasil, é de suma relevância e urgência o desenvolvimento de agentes antichágasicos realmente eficazes, principalmente para a fase crônica da doença (JANNIN \& VILLA, 2007). Esses compostos, nifurtimox e benznidazol, apresentam ainda outros inconvenientes (Tabela 1), tais como: (a) graves e frequentes efeitos colaterais como vômito, anorexia, neuropatia periférica, dermopatia alérgica, alterações psíquicas, mutagenicidade, entre outras; (b) sensibilidade seletiva para diferentes cepas de . cruzi; (c) tratamentos muito longos (JANNIN \& VILLA, 2007; KOROLKOVAS, 2002). 
Tabela 1 - Principais efeitos colaterais observados no tratamento específico da doença de Chagas.

\begin{tabular}{lll}
\hline \hline Sint oma / Sinal & Benznidazol & Nifurtimox \\
\hline \hline Anorexia & ++ & +++ \\
Cefaléia & + & ++ \\
Dermatopatia & +++ & + \\
Excitação psíquica & - & +++ \\
Gastralgia & + & +++ \\
Insônia & + & ++ \\
Náusea & ++ & +++ \\
Perda de peso & + & +++ \\
Polineuropatia & + & ++ \\
Vômito & ++ & +++ \\
\hline \hline
\end{tabular}

Fonte: Cançado (1997), Rassi et al. (2000).

Novos derivados 5-nitrofurânicos, semicarbazonas (VIII), carbazatos (XI), e amidas (X) (Figura 2) mostraram excelente atividade tripanomicida: a presença de cadeias apolares em cada família dos derivados resultou em compostos mais ativos do que aqueles com substituintes menos apolares. Os derivados de carbazatos apresentaram maior atividade que os de semicarbazonas, sendo o heptil carbazato (IX) o composto mais potente (AGUIRRE et al., 2006), enquanto a presença do grupo apolar adamantil (III), confere a este composto uma melhor atividade tripanomicida.

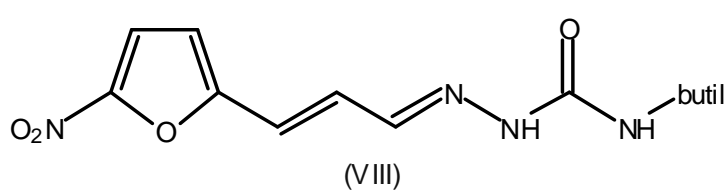<smiles>CCCCCC(=O)N/N=C/C=C/c1ccc([N+](=O)[O-])o1</smiles><smiles>O=C(/C=C/c1ccc([N+](=O)[O-])o1)NC12CC3CC(CC(C3)C1)C2</smiles>

Figura 2 - Estrutura química dos compostos semicarbazona (VIII), heptilcarbazato (IX) e adamantil (X), sintetizados por Aguirre et al., 2006.

O experimento de Otero et al. (2006) mostra que complexos derivados de nitrofurilsemicarbazona com rênio (XI) ou rutênio (XII) (Figura 3) podem ser um bom ponto de partida no planejamento de novos compostos com atividade contra T. cruzi, pois aumentam o estresse oxidativo e os níveis de ligação com o DNA. 

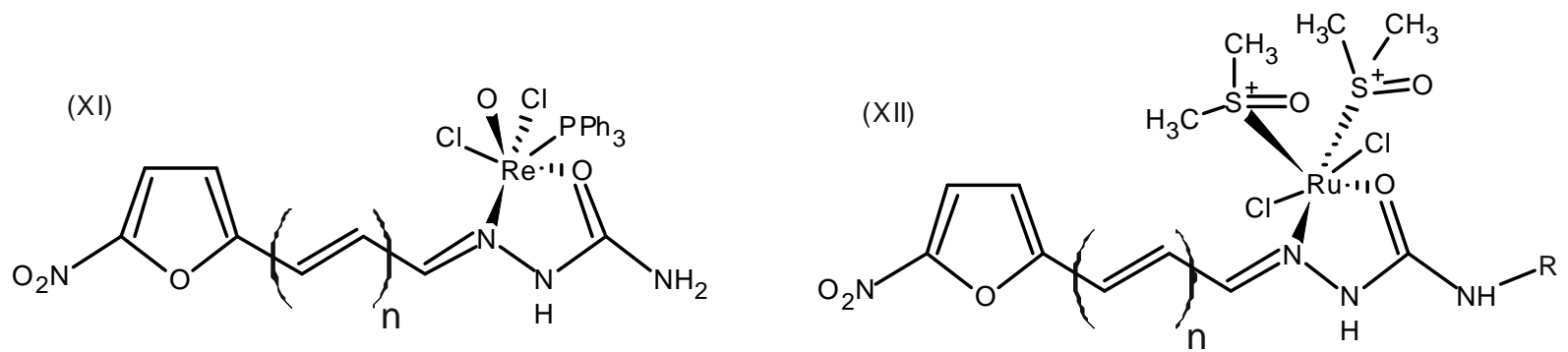

Figura 3 -Estruturas químicas dos compostos derivados de nitrofurilsemicarbazona com rênio (XI) e rutênio (XII) desenvolvidos por Otero et al. (2006).

Em outro experimento, Otero e colaboradores (2006) desenvolveram complexos derivados de nitrofuriltio semicarbazona com paládio (XIII) (Figura 4). Tais compostos, em testes in vitro, se mostraram mais ativos que o nifurtimox. O efeito tripanomicida pode ser explicado pelo principal mecanismo de ação desses compostos, os quais estão relacionados como aumento do estresse oxidativo.<smiles>CCC=CC=CNN1NC(NP)=[SH]C1(Cl)Cl</smiles>

Figura 4 -Estrutura química de complexos de nitrofuriltio semicarbazona com paládio.

Boiani e colaboradores (2006) desenvolveram diversos compostos derivados de benzimidazóis com atividade in vivo contra o T. cruzi.<smiles>CC1(C)[N+]([O-])=c2ccc(/C=N/O)cc2=[N+]1[O-]</smiles>

(XIV)
Entre eles aparecem os compostos mais promissores, com baixa toxicidade, apresentados na Figura 5.<smiles>CC1(C)[N+]([O-])=c2ccc(C3OCCO3)cc2=[N+]1[O-]</smiles>

Figura 5 - Estrutura química dos principais derivados de benzimidazóis (XIV) e (XV) com atividade contra T. cruzi (BOIANI et al., 2006).

O estudo de fármacos mais específicos, isto é, seletivos ao tripanosoma e, consequentemente, menos tóxicos ao homem, tem sido o objetivo principal do desenvolvimento de novos quimioterápicos (WOLFF, 1995). Enzimas específicas, presentes apenas nos tripanossomos, estão sendo estudadas como alvos. 
Entre os alvos quimioterápicos potenciais em T. cruzi encontram-se a tripanotiona redutase (KRAUTHSIEGEL \& INHOFF, 2003; LOCKMAN \& HAMILTON, 2005; OLIVEIRA et al., 2006; PRIETO \& TALEVI \& BRUNO-BLANCH, 2006; RAVASCHINO \& DOCAMPO \& RODRIGUEZ, 2006; IRIBARNE et al., 2007), a glutationa redutase GR(IRIBARNE et al., 2007), a cruzipaína ou GP 57/51 (CAZZULO, 2002; LOCKMAN \& HAMILTON, 2005; BRAK et al., 2008), serina, treonina e metaloproteinases (CAZZULO, 2005), a GAPDH (gliceraldeído-3-fosfato desidrogenase) (ZACKS, 2006) e outras enzimas glicolíticas (VERLINDE et al., 2001), a transialidase (AGUSTÍ et al., 2004; WORONOWICZ et al., 2004; DUTHIE et al., 2005), a biossíntese de purinas (FREYMANN et al., 2000), o metabolismo de folato e pteridina (LOCKMAN \& HAMILTON, 2005), a biossíntese do RNAm (BARBOSA et al., 2007), a biossíntese do ergosterol (BUCKNER et al, 2003; URBINA et al., 2003), dUTP nucleotideohidrolase (HARKIOLAKI et al, 2004), o cinetoplasto (SCHNARE \& GRAY, 1999), DNA topoisomerase de tripanosomatídeos (BALAÑA-FOUCE et al., 2006), $\alpha$-hidroxiacidodesidrogenase isoenzima II (CHENA et $a l .$, 2004), oleato desaturase (MALDONADO et al., 2006) e DHOD (diidrooronato desidrogenase) (NARA et al., 2005), C-14 $\alpha$ desmetilase (BUCKNER et al., 2003; URBINA et al., 2003).

A mutagenicidade e a possível carcinogenicidade dos nitrofuranos devem-se, provavelmente, à presença de dois grupos potencialmente reativos: o nitro na posição 5 e os substituintes (R) na posição 2 do anel furânico (MCCALLA, 1983; KOBIERSKASZELIGA \& CZECZOT, 1994; MECCA \& DIAZ \& CASTRO, 2002; HIRAKU et al., 2004; DAVIES et al., 2010).

O esquema apresentado na Figura 6 mostra os mecanismos, propostos na literatura, de toxicidade e mutagenicidade dos nitrofurânicos (MCCALLA, 1983; MECCA \& DIAZ \& CASTRO, 2002; HIRAKU et al., 2004). Em sistemas bacterianos, o requisito básico para a ação mutagênica de compostos nitrofurânicos é a redução promovida por, no mínimo, três nitrorredutases. Essas reduções resultam em cadeia de reações, que levam à formação de espécies eletrofilicas que podem reagir com o DNA.

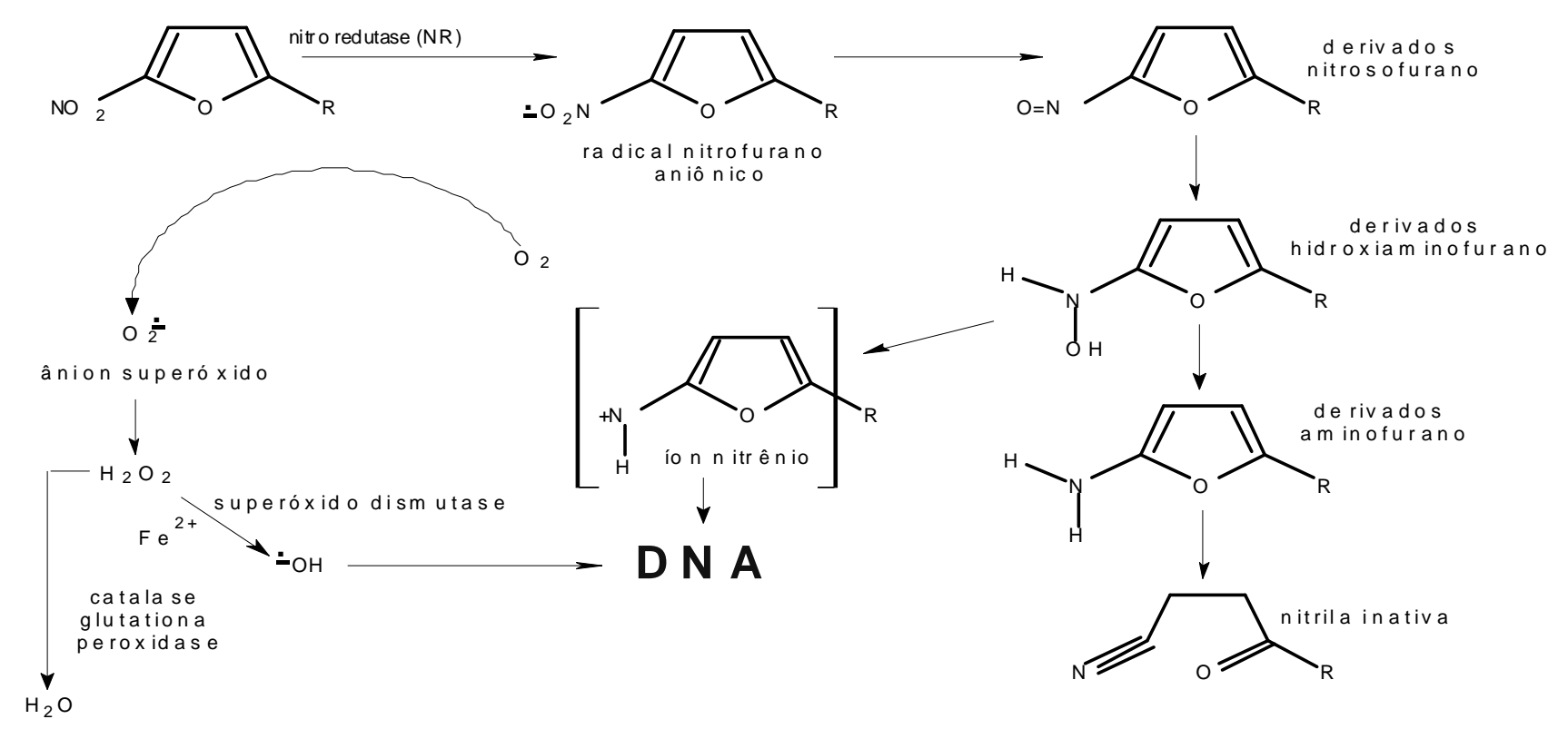

Figura 6 - Possíveis mecanismos de mutagenicidade dos nitrofurânicos.

Fonte: Adaptado de McCalla (1983), Mecca \& Diaz \& Castro (2002); Hiraku et al. (2004). 
A completa redução desses compostos envolve a adição de seis elétrons para formar a amina via o

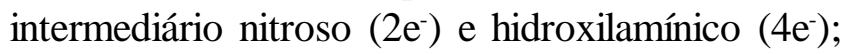
entretanto alguns fármacos não prosseguem, além da formação da hidroxilamina (TOCHER, 1997). O alvo desses compostos é o DNA; a redução dos mesmos causa quebra e desestabilização da sua hélice (EDWARDS, 1993). O grau do dano está relacionado com a composição da fita e aumenta com a presença de adenina (A) e timina (T) no DNA. Aidentificação do agente causador do dano não está bem elucidada, já que os produtos finais da redução desses compostos são inativos, mas a redução dos mesmos na presença do DNA resulta em dano, sugerindo que esse é causado pelos pequenos intermediários formados pela adição de no mínimo quatro elétrons. Paralelamente, ocorre a abertura do anel nitrofurânico, formando derivado nitrila inativo (MCCALLA, 1983; KOBIERSKA-SZELIGA \& CZECZOT, 1994). A Figura 7 mostra a redução desses compostos (TOCHER, 1997).

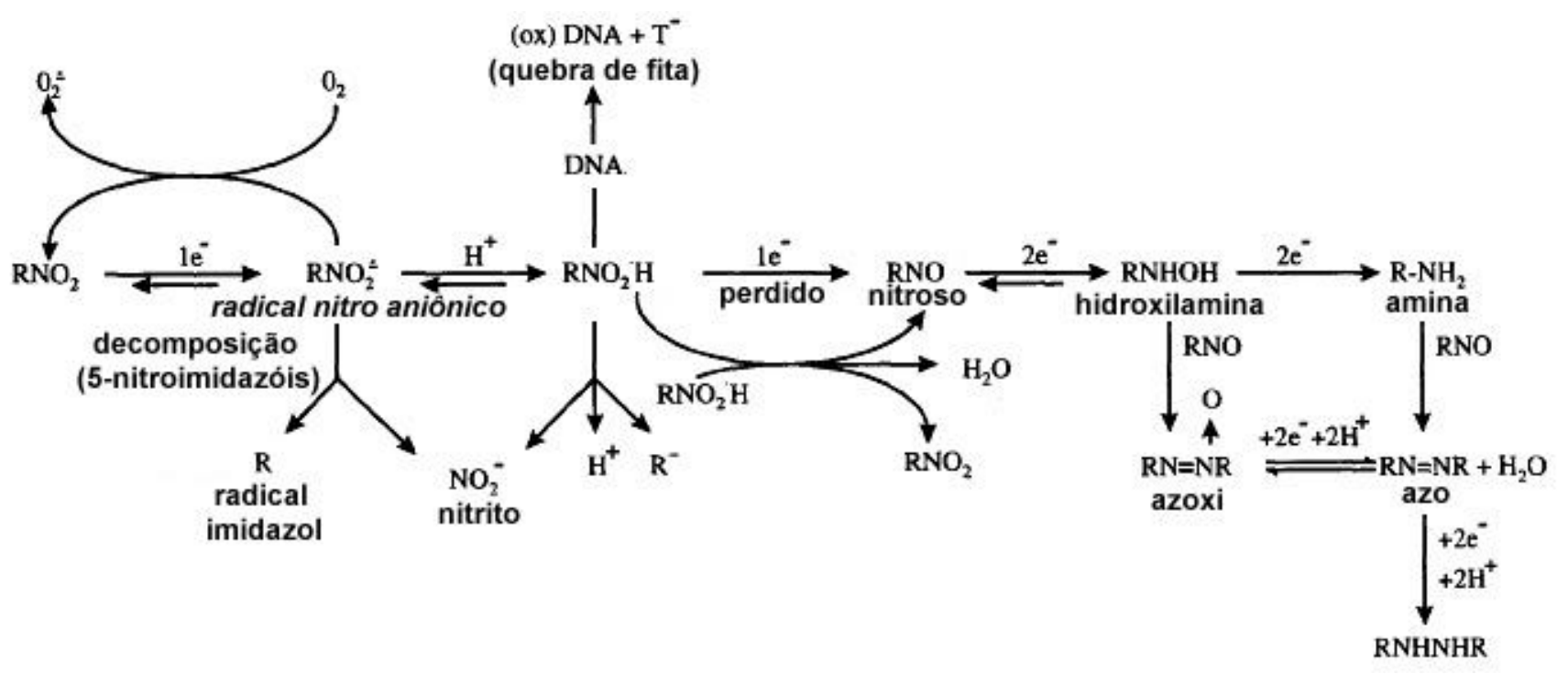

Figura 7 - Ativação por redução de compostos nitroaromáticos (TOCHER, 1997).

Estudo de relações quantitativas entre estrutura e atividade (QSAR) sobre 2-nitrocompostos aromáticos, utilizados como agentes antibacterianos, demonstraram que o aumento da densidade eletrônica no carbono da posição 2 do anel aromático aumenta o efeito mutagênico. Possivelmente, a maior densidade eletrônica nesse carbono deva estabilizar o anel furânico e aumentar a probabilidade para a reação com o DNA (DEBNATH et al., 1993).

O trabalho de revisão de Stoppani e colaboradores (1996) descreve o papel das espécies de oxigênio ativo na quimioterapia da doença de Chagas, incluindo as propriedades eletrônicas de novos compostos em relação à capacidade de produzir radicais nitroaniônicos e a respectiva atividade tripanomicida.

\section{Planejamento de novos fármacos}

O processo de descoberta de novos fármacos, também uma atribuição da Química Farmacêutica e Medicinal, caracteriza-se por sua complexidade, fruto da multiplicidade de fatores que envolvem o planejamento molecular de novas estruturas capazes de apresentar os efeitos farmacológicos desejados, com emprego terapêutico seguro (BARREIRO \& FRAGA, 2001; DIAS et al., 2009; SANTOS \& MATOS \& FERREIRA, 2011). Ainda hoje, existem diversos fármacos (alguns muito potentes), cujas características físico-químicas, organolépticas, farmacocinéticas, farmacológicas e toxicológicas são barreiras para sua aplicação clínica (ZHENG et al., 1999; HAN \& AMIDON, 2000; TESTA\& MAYER, 2003 ). 
Para otimizar as características físico-químicas de um fármaco, podem-se derivar certos grupos funcionais polares com pequenas moléculas orgânicas biorreversíveis, mascarando tais características sem alterar permanentemente as propriedades da molécula. Tal estratégia tem sido utilizada com sucesso e grupos funcionais, tais como álcoois convertidos emésteres, os quais podem ser rapidamente convertidos in vivo por via química ou enzimaticamente (ZHENG et al., 1999).

Essa estratégia para a superação dos problemas anteriormente referidos e para a busca de novos compostos terapêuticos é a latenciação de fármacos, em que o termo latente significa: presente ou existente, mas não manifestada, exibida ou desenvolvida (CHUNG et al., 2005).

A latenciação de fármacos foi proposta, em 1959, por Harper, a qual consiste na transformação do fármaco em forma de transporte inativo que, in vivo, mediante reação química ou enzimática, libera a porção ativa no local de ação ou próximo dele. Entretanto, somente em meados da década de 1970, quando pesquisadores começaram a localizar os alvos dos fármacos no organismo e compreender a farmacocinética dos mesmos, é que o processo de latenciação tomou uma direção mais definida (HAN \& AMIDON, 2000; CHUNG et al., 2005). Nos últimos anos, a latenciação tornou-se uma das principais ferramentas no desenvolvimento de novos quimioterápicos, para o combate de enfermidades como o câncer e a aids (CHUNG et al., 2005).

O fármaco latente é uma espécie de "Cavalo de Troia", uma vez que este engana o organismo, não para destruí-lo, mas para ajudá-lo. Tanto o fármaco latente quanto o análogo possuem estruturas químicas similares, mas as propriedades biológicas desses compostos diferem em relação à do fármaco matriz quanto: à atividade, à potência, à biodisponibilidade, à síntese, ao espectro de ação, ao índice terapêutico, entre outros. Um análogo, muitas vezes, difere do fármaco protótipo em um só átomo ou em um grupo de átomos. Todavia, estruturalmente, o fármaco protótipo e o análogo possuem características próprias, oriundas de sua estrutura química (Figura 8). 


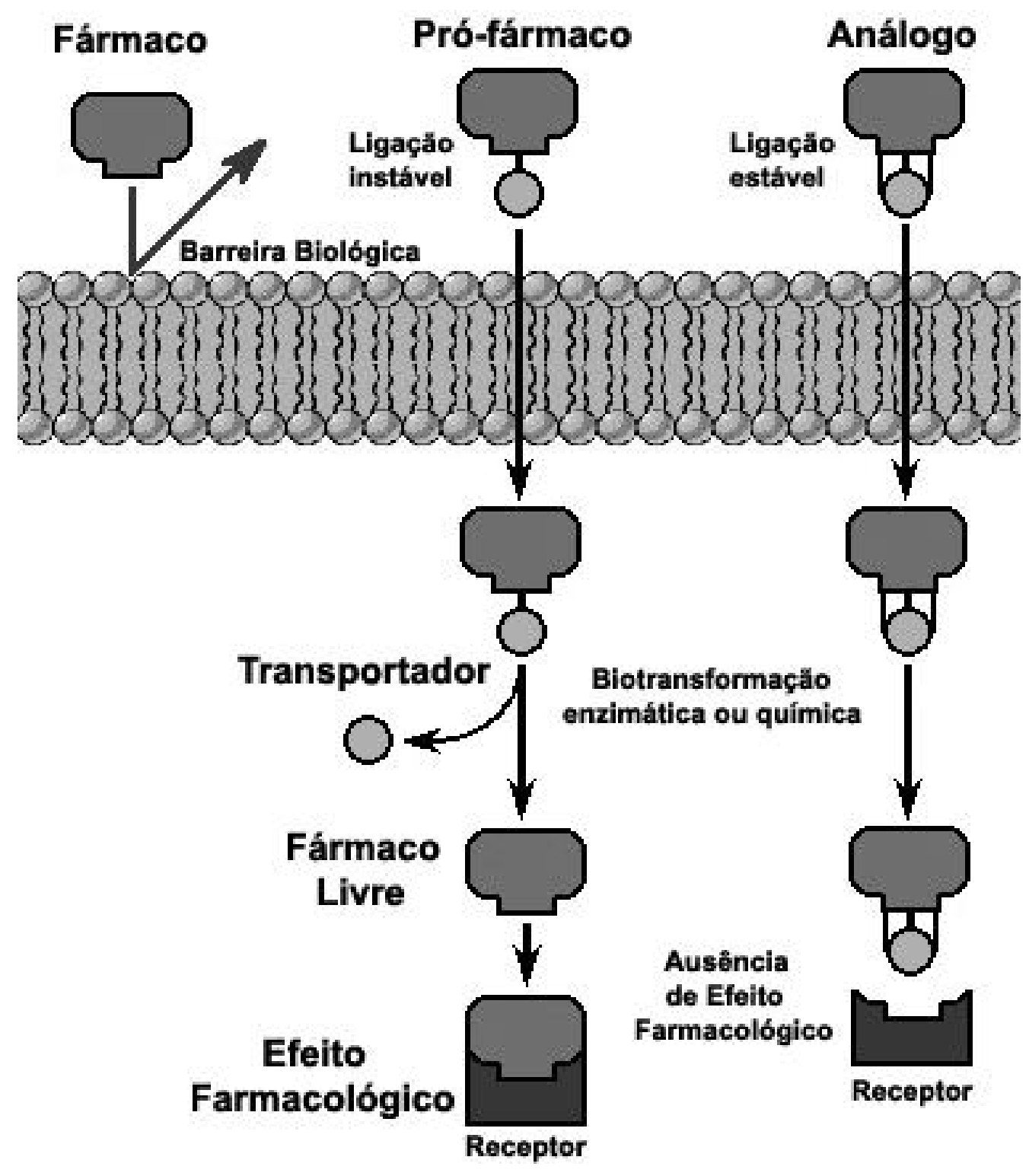

Figura 8 - Diferenças entre Fármaco, Pró-fármaco e Análogo (CHUNG et al., 2005).

Muitos problemas relacionados ao fármaco matriz justificam a busca por novos fármacos latentes, tais como: problemas farmacocinéticos, alta toxicidade, baixa estabilidade química, solubilidade inapropriada, odor e paladar indesejáveis, dor no local da administração e formulação farmacêutica de difícil preparo. Os principais problemas farmacocinéticos descritos por Bundgaard (1991) incluem: deficiência de biodisponibilidade oral (coeficiente de partição inadequado e/ou hidrossolubilidade); distribuição inespecífica e incapacidade de atravessar diversos tipos de barreiras biológicas (mucosa gástrica, pele, córnea e barreira hematoencefálica), que separam o fármaco de seu local de ação, entre outros.

As formas latentes de fármacos podem ser classificadas, de acordo com Wermuth (2004), em pró-fármacos clássicos, bioprecursores, fármacos dirigidos e pró-fármacos mistos. Uma definição mais 
abrangente considera um pró-fármaco como um fármaco ativo, quimicamente transformado em um derivado inativo, o qual é convertido no fármaco matriz dentro do organismo antes ou após alcançar seu local de ação por um ataque químico ou enzimático ou de ambos (CHUNG et al., 2005). O desenvolvimento de pró-fármacos tem como objetivo resolver diversos problemas relacionados aos fármacos atuais: alterar a farmacocinética do fármaco in vivo, para melhorar sua absorção, distribuição, biotransformação e excreção; diminuir sua toxicidade e os efeitos adversos; aumentar sua especificidade e tempo ação e sua solubilidade e estabilidade.

Os pró-fármacos possuem alguns fatores importantes em seu desenvolvimento, para permitir o aprimoramento das propriedades do fármaco matriz, tais como: ser inativo; sua síntese deve ser menos complexa que a do fármaco matriz; a ligação entre o fármaco matriz e o transportador deve ser cindida, in vivo, por via química ou enzimática; o transportador não deve ser tóxico; deve também possuir cinética adequada de biotransformação direta tanto quanto de inativação, assegurando níveis eficazes do fármaco no local de ação.

A vantagem do desenvolvimento de pró-fármacos é a facilidade de obtenção de novos compostos, não considerados "me too" e, portanto passível de patentes (CHUNG et al., 2005; SILVA et al., 2005). Nesse contexto, diversos pró-fármacos foram inseridos na terapêutica. No mercado alemão, 6,9\% dos fármacos comercializados são pró-farmacos. Entre eles se destacam substâncias como: enalapril, omeprazol, sinvastatina e aciclovir, considerados blockbusters devido ao potencial mercadológico (ETTMAYER et al., 2004). Diversas técnicas metodológicas foram descritas na literatura (KUBINYI,1995a; BÖHM et al., 2000), permitindo avanços significativos no planejamento e desenvolvimento de novos fármacos, tais como: química combinatória (GORDON \& KERWIN, Jr., 1998), engenharia genética (MEYER, 1996), cristalografia de proteínas (RONDEAU \& SCHREUDER, 1996), técnicas multidimensionais em RMN (SCHUKER et al., 1996) relações quantitativas entre estrutura química e atividade biológica-QSAR (HANSCH \& FUJITA, 1964; FUJITA, 1990; KUBINYI, 1993a, 1995; SEYDEL, 1979) e a variante QSAR-3D, modelagem molecular, planejamento dirigido com base na estrutura e auxiliado por computador, CADD (KUBINYI, 1993b,1995a,1995b; KLEBE \& ABRAHAM, 1999; SCHUKER \& HADJUC, 1996; BÖHM, 1993; BÖHM et al., 2000; AGUIRRE et al., 2006), e também planejamento de 4D-QSAR ( LIU et al., 2003; PITA et al., 2006), entre outras.

No caso de antichagásicos, várias substâncias são projetadas somente contra a forma epimastigota, embora alguns autores já relatem sensibilidades diferentes a fármacos entre formas diferentes do parasito. Em relação aos testes in vivo, uma padronização de modelos biológicos em ambas as fases, aguda e crônica, e de parâmetros da cura é de suma importância (COURA \& CASTRO, 2002).

Segundo Croft e colaboradores (2005), novos compostos antichagásicos (Figura 9) estão em fase préclínica, como: triazóis (ravuconazol (XVII) e TAK-187 (XVIII); o inibidor irreversível (K-777) (XIX) da cruzipaína, uma enzima (protease) essencial presente em todos os estágios do T. cruzi e que possui atividade in vitro e in vivo; novos derivados triazóis, como o D-0870 (XX), que são inibidores da esterol C14 $\alpha$ desmetilase do T. cruzi, possuindo atividade potente e seletiva, erradicando o parasita em modelos animais em ambas as fases da doença de Chagas (aguda e crônica), estando atualmente em desenvolvimento como agentes antifúngicos sistêmicos. 

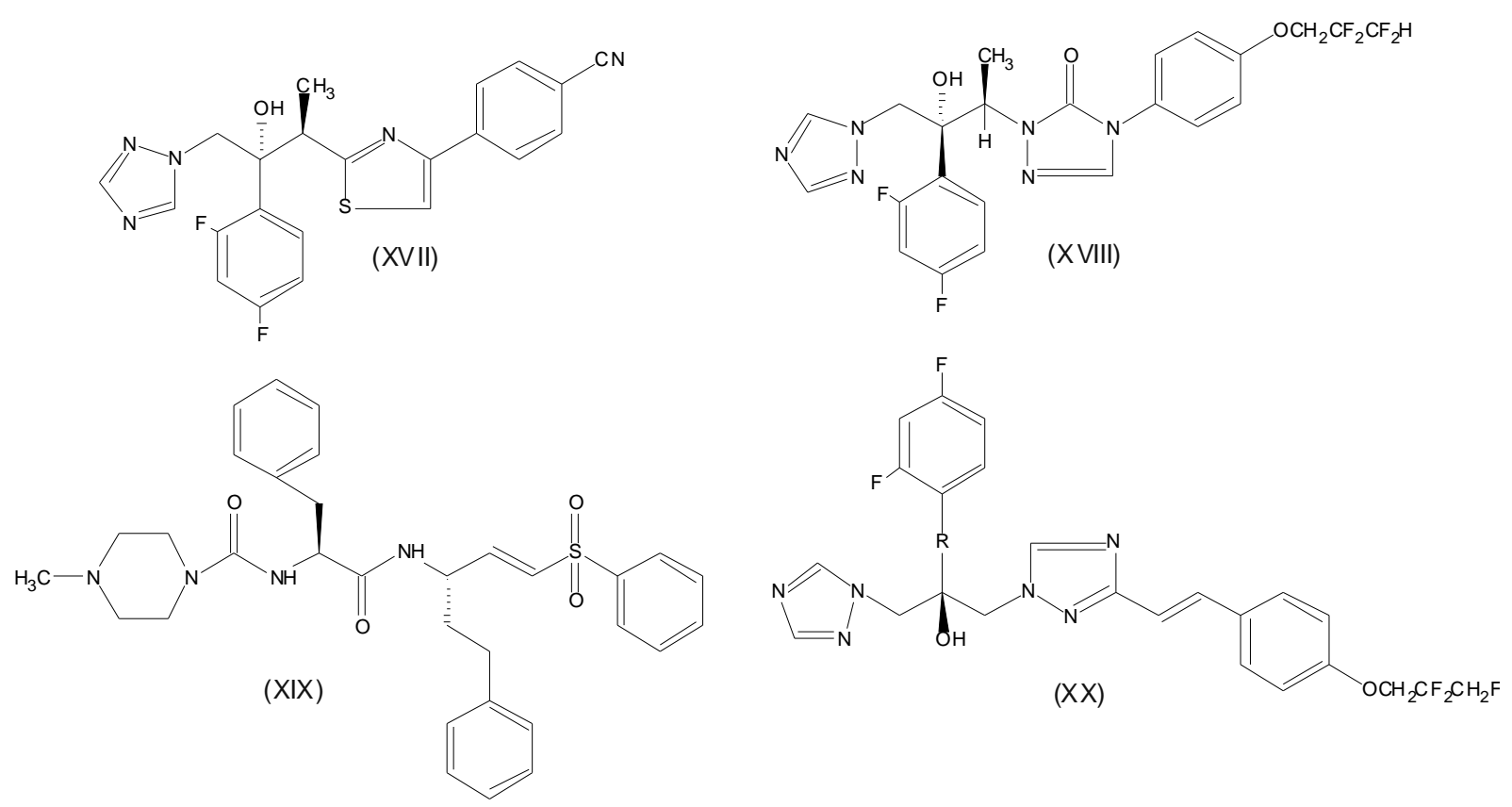

Figura 9 - Estrutura química de novos compostos antichagásicos: (XVII) ravuconazol, (XVIII) TAK-187, (XIX)) K-777 e (XX) D-0870 (CROFT \& BARRETT \& URBINA, 2005).

Aguirre e colaboradores (2005) desenvolveram 26 compostos derivados de benzofuroxanas, entre eles, o composto (I), que originou diversos análogos com excelente atividade in vitro, contra a forma epimastigota do T. cruzi, sendo o composto (II) o mais eficiente. Também foi verificado que, quando se retira o grupo fenil desses análogos, ocorre a perda de atividade (Figura 10).<smiles></smiles><smiles>[R]C=Cc1ccc2nonc2c1</smiles>

Figura 10 - Estrutura química dos compostos antichagásicos (XXI) e (XXII) (AGUIRRE et al., 2005).

Outros compostos, descritos por Souza e colaboradores (2005), demonstraram atividade antichagásica em testes in vitro contra as formas tripomastigota e amastigota, entre os quais (-) metilpluviatolida e (-) cubebina, mas não in vivo.

Embora haja um empenho pela busca por medicamentos para a doença de Chagas, a Drugs for Neglected Diseases Initiative - DNDi (2011), em seu portfólio, indica ainda um número pequeno de novos agentes antichagásicos, tais como: benzofuroxanas, inibidores de tripanotiona redutase (TR), inibidores de diidrofolato redutase (DHFR), nitroimidazóis, sendo que o benznidazol pediátrico e os azóis E1224 \& biomarker já estão em fase clínica.

Dentre os compostos potencialmente antichagásicos em estudo, o hidroximetilnitrofural (NFOH), um prófármaco derivado do nitrofural, mostrou maior atividade tripanomicida e menor mutagenicidade do que o nitrofural (composto matriz) em todos os estágios de desenvolvimento de um fármaco. 
$\mathrm{O} \mathrm{NFOH}$ apresenta estabilidade in vitro, e é metabolizado em plasma (humano e de ratos) e também, provavelmente, pelo fígado. $\mathrm{O} \mathrm{NFOH}$, através dos estudos realizados, aliado à estabilidade química favorecida pela sua estrutura, é um potencial candidato a fármaco antichagásico para os estudos clínicos (SERAFIM, 2008; DAVIES et al., 2010; SANTOS; MATOS; FERREIRA, 2011).

\section{Considerações Finais}

Mais de 1 bilhão de pessoas na América Latina, Africa e Ásia são ainda afetadas por pelo menos 1 das 17 doenças que estão na lista pela Organização Mundial da Saúde - OMS (WHO na sigla em inglês) como doenças tropicais negligenciadas - NTDs. Adoença de Chagas, incluída entre as negligenciadas, tornou-se mais do que simplesmente uma doença zoonótica que afeta principalmente a população rural, sendo uma preocupação mundial que pode ter consequências epidemiológicas, econômicas, sociais e políticas. Diversas técnicas metodológicas vêm sendo descritas na literatura, permitindo avanços significativos no planejamento e desenvolvimento de novos agentes antichagásicos, com ênfase na busca de pró-fármacos que permitam o aprimoramento do fármaco matriz. Estudos sobre compostos antichagásicos devem ser incentivados pela comunidade científica e representam um desafio para os pesquisadores da área, particularmente para o tratamento da fase crônica da doença.

\section{REFERÊNCIAS}

AGUIRRE, G. L.; BOIANI, L.; BOIANI, M.; CERECETTO, H.; DI MAIO, R.; GONZÁLEZ, M.; PORCAL, W.; DENICOLA, A.; PIRO, O. E.; CASTELlANO, E. E.; SAN'ANNA, C. M. R.; BARREIRO, E. J. New potent 5-substituted benzofuroxans as inhibitors of Trypanosoma cruzi growth: Quantitative structure-activity relationship studies. Bioorganic \& Medicinal Chemistry, v. 13, p. 6336-6346, 2005.

AGUIRRE, G.; BOIANI, M.; CABRERA, E.;
CERECETTO, H.; DI MAIO, R.; GONZÁLEZ, M.; DENICOLA, A.; SANT'ANNA, C. M. R.; BARREIRO, E. J. New potent 5-nitrofuryl derivatives as inhibitors of Trypanosoma cruzi growth. 3D-QSAR (CoMFA) studies. European Journal of Medicinal Chemistry, v. 41, n. 4, p. 457-466, 2006.

AGUSTÍ, R.; PARÍS, G.; RATIER, L.; FRASCH, A.; C. C.; LEDERKREMER, R. M. Lactose derivatives are inhibitors of Tripanosoma cruzi trans-sialidase activity toward conventional substrates in vitro and in vivo. Glycobiology, v. 14, n. 7, p. 659-670, 2004.

\section{AMATO-NETO, V, PASTERNAK, J.}

Chagas'disease centenary. Rev. Saúde Pública, v. 43, p. 381-382, 2009.

ANANDAN, J. V. Parasitic disease. In: DIPIRO, J. T. (Ed.). Pharmacotherapy: a pathophysiologic approach. 3. ed. London: Appleton \& Lange, 1997. p. 2161-2172.

BALAÑA-FOUCE, R.; REDONDO, C. M.; PERTEJO, Y. P.; GONZÁLEZ, R. D.; REGUERA, R. M. Targeting atypical trypanosomatid DNA topoisomerase I. Drug Discovery Today, v.11, n. 15/16, p. 733-740, 2006.

BARBOSA, C. F.; OKUDA, É. S.; CHUNG, M. C.; FERREIRA, E. I.; CICARELLI, R. M. B.; BARBOSA, C. F. Rapid test for the evaluation of the activity of the prodrug hydroxymethylnitrofurazone in the processing of Trypanosoma cruzi messenger RNAs. Brazilian Journal of Medical Biological Research, v. 40, n. 1, p. 33-39, jan. 2007.

BARREIRO, E. J.; FRAGA, C. A. M. Química medicinal: as bases moleculares da ação dos fármacos. Porto Alegre: ARTMED, 2001.

BÖHM, H. J. Ligand design. In: KUBINYI, H. 3D 
QSAR in Drug Design: theory, methods and applications. Leiden: ESCOM, 1993. p. 386-405.

BÖHM, H. J.; BOERINGER, M.; BUR, D.; GMUENDER, H.; HUBER, W.; KLAUS, W.; KOSTREWA, D.; KUEHNE, H.; LUEBBERS, T.; MEUNIERKELLER, N.; MUELLER, F. Novel inhibitors of DNA gyrase: 3D structure based biased needle screening, hit validation by biophysical methods, and 3D guided optimization: a promising alternative to random screening. Journal of Medicinal Chemistry, v. 43, n. 14, p. 2664-2674, 2000.

BOIANI, M.; BOIANI, L.; DENICOLA, A.; ORTIZ, S. T.; SERNA, E.; BILBAO, N. V.; SANABRIA, L.; YALUFF, G.; NAKAYAMA, H.; ARIAS, A. R.; VEJA, C.; ROLAN, M.; GÓMEZBARRIO, A.; CERECETTO, H.; GONZÁLEZ, M. 2H-Benzimidazole 1,3-Dioxide Derivatives: a new family of water-soluble anti-trypanosomatid agents. Journal of Medicinal Chemistry, v. 49, n. 11, p. 3215-3224, 2006.

BRAK K,DOYLE, PS, MACKERROW, JH, ELLMAN, JA. Identification of a new class of nonpeptidic inhibitors of cruzain. Journal of the American Chemical Society, v.130, p.6404-10, 2008.

BRENER, Z.; CANÇADO, J. R. Terapêutica. In: BRENER, Z.; ANDRADE, Z. A. Trypanosoma cruzi e Doença de Chagas. Rio de Janeiro: Guanabara Koogan, 1979. p. 362-424.

BUCKNER, F.; YOKOYAMA, K.; LOCKMAN, J.; AIKENHEAD.; OHKANDA, J.; SADILEK, M.; SEBTI, S.; VOORHIS, W. V.; HAMILTON, A.; GELB, M. Aclass of sterol 14-demethylase inhibitor as anti-Trypanosoma cruzi agents.

Proceedings of the National Academy of Sciences of the United States of America, v. 100, n. 25, p. 15149-15153, 2003.
BUNDGAARD, H. Novel chemical approaches in prodrug design. Drugs of the Future, v. 16, n. 5, p. 443-458, 1991.

CANÇADO, J. R. Terapêutica específica. In: DIAS, J. C. P.; COURA (Org.). Clínica e terapêutica da doença de Chagas: uma abordagem prática para o clínico geral. Rio de Janeiro: FIOCRUZ, 1997. p. 323-352.

CAZZULO, J. J. Proteinases of Trypanosoma cruzi: potential targets for the chemotherapy of Chagas disease. Current Topics in Medicinal Chemistry, v. 2, n. 11, p. 1261-1271, 2002.

CAZZULO, J. J. Proteinases of Trypanosoma cruzi: potential targets for the chemotherapy of Chagas disease. Medicinal Chemistry Reviews, v. 2, n. 6, p. 495-504, 2005.

CHENA, M. A.; JIMÉNEZ, S. E.; PÁEZ, L. R.; TORRES, B. N.; RAMÍREZ, I. B.; RAMÍREZ, C. W. Trypanosoma cruzi: Inhibition of -hydroxyacid Dehydrogenase Isozyme II by N-allyl and N-propyl oxamates and their Effects on Intact Epimastigotes.

Memórias do Instituto Oswaldo Cruz, v. 99, n. 8, p. 831-837, 2004.

CHUNG, M. C.; SILVA, A. T. A.; CASTRO, L. F. et al. Latentiation and advanced drug transport forms. Revista Brasileira de Ciências Farmaceuticas, v. 41, n. 2, p. 155-180, 2005.

COURA, J. R.; CASTRO, S. L. A critical review on chagas disease chemotherapy. Memórias do Instituto Oswaldo Cruz, v. 97, n. 1, p. 3-24, jan. 2002.

COURA,J.R.; VINÃS, P.A. Chagas disease: a new worldwide challenge. Nature, v.465, p.56-57, 2010.

CROFT, S. L.; BARRETT, M. P.; URBINA, J. A. 
Chemotherapy of trypanosomiases and leishmaniasis. Trends in Parasitology, v. 21, n. 11, p. 508-512, 2005.

DAVIES, C, CARDOZO, R.M, NEGRETTE, O.S, MORA, M. C, CHUNG, M.C, BASOMBRIO, M.A. Hydroximethylnitrofurazone is active in a murine modela of Chagas's Disease. Antimicrob. Agents Chemother, v. 54, p. 3584-3589, 2010.

DEBNATH, A. K.; HANSCH, C.; KIM, K. H.; MARTIN, Y. C. Mechanistic interpretation of the genotoxicity of nitrofurans (antibacterial agents) using quantitative structure-activity relationships and comparative molecular field analysis. Journal of Medicinal Chemistry, v. 36, n. 8, p. 1007-1016, 1993.

DIAS, J. C. P. Doença de Chagas: clínica e terapeutica. Brasília: Ministério da Saúde: SUCAM, 1990.

DIAS, J. C. P. Epidemiologia. In: BRENER, Z.; ANDRADE, Z. A.; BARRAL NETTO, M. Trypanosoma cruzi. 2.ed. Rio de Janeiro: Guanabara Koogan, 2000. p. 48-74.

DIAS, L. C.; DESSOY, M. A.; SILVA, J. J. N., THIEMANN, O. H.; OLIVA, G.;

ANDRICOPULO, A. D. Quimioterapia da doença de Chagas: estado da arte e perspectivas no desenvolvimento de novos fármacos. Quim. Nova. v. 32, n. 9, p. 2444-2457, 2009.

\section{DRUGS FOR NEGLECTED DESEASES} INICIATIVE. DNDI R\&D Projects. 2011. Disponível em: <http://www.dndi.org/index.php/ portfolio.html?ids=2> . Acesso em: 12 ago. 201.1

DRUMOND, J. A. G.; MARCOPITO, L. F. Migração interna e a distribuição da mortalidade por doença de Chagas, Brasil, 1981/1998.

Cadernos de Saúde Pública, v. 22, n.10, p.
2131-2140, 2006.

DUTHIE, M. S.; CETRON, M. S.; VAN

VOORHIS, W. C.; KAHN, S. J. Trypanosoma cruzi-infected individuals demonstrate varied antibody responses to a panel of trans-sialidase proteins encoded by SA85-1 genes. Acta Tropica, v. 93 , n. 3, p. 317-329, 2005.

EDWARDS, D. I. Nitroimidazole drugs - ction and resistance mechanisms. I. Mechanisms of action. Journal of Antimicrobial Chemotherapy, v. 31, n. 1, p. 9-20, 1993.

ETTMAYER, P.; AMIDON, G. L.; CLEMENT, B.; TESTA, B. Lessons learned from marketed and investigational prodrugs. Journal of Medicinal Chemistry, v. 47, n. 10, p. 2393-2404, 2004.

FREYMANN, D. M.; WENCK, M. A.; ENGEL, J. C.; FENG, J.; FOCIA, P. J.; EACKIN, A. E.; CRAIG, S. P. Efficient identification of inhibitors targeting the closed active site conformation of inhibitors of the HPRT from Trypanosoma cruzi. Chemistry and Biology, v. 7, p. 957-968, 2000.

FUJITA, T. The extrathermodynamic approach to drug design, In: HANSCH. C.; SAMMES, P. G.; TAYLOR, J. B. (Ed). Comprehensive medicinal chemistry: the rational design, mechanistic study \& therapeutic application of chemical compounds. Oxford: Pergamon, 1990. v. 4, p. 497-560.

GORDON, E. M.; KERWIN JR., J. F. (Ed.). Combinatorial chemistry and molecular diversity in drug discovery. New York: WileyLiss, 1998.

HAN, H. K.; AMIDON, G. L. Targeted prodrug design to optimizedrug delivery. AAPS

Pharmaceutical Science, v. 2, p. E6, 2000.

HANSCH, C.; FUJITA, T. Analysis: a method for 
correlation of biological activity and chemical structure. Journal of American Chemical Society, v. 86, n. 8 , p. 1616-1626, 1964.

HARKIOLAKI, M.; DODSON, E. J.; BERNIER-VILLAMOR, V.; TURKENBURG, J. P.; GONZÁLEZ-PACANOWSKA, D.; WILSON, K. S. The crystal structure of Trypanosoma Cruzi dUTPase reveals a novel dUTP/ dUDP binding fold. Structure, v. 12, n. 1, p. 41-53, 2004.

HIRAKU, Y.; SEKINE, A.; NABESHI, H.; MODORIKAWA, K.; MURATA, M.; KUMAGAI, Y.; KAWANISHI, S. Mechanism of carcinogenesis induced by a veterinary antimicrobial drug, nitrofurazone, via oxidative DNA. Cancer Letters, v. 215, n. 2, p. 141-150, 2004.

IRIBARNE, F.; GONZÁLEZ, M.; CERECETTO, H.; AGUILERA, S.; TAPIA, O.; PAULINO, M. Interaction energies of nitrofurans with trypanothione reductase and glutathione reductase studied by molecular docking. Journal of Molecular Structure: THEOCHEM, v. 818, n. 1/ 3, p. 7-22, 2007.

JANNIN, J.; VILLA, L. An overview of Chagas disease treatment. Memórias do Instituto Oswaldo Cruz, v. 102, p. 95-97, jul. 2007. Suplemento I.

KLEBE, G.; ABRAHAM, U. Comparative molecular similarity index analysis (CoMSIA) to study hydrogen-bonding properties and to score combinatorial libraries. Journal of ComputerAided Molecular Design, v. 13, n. 1, p. 1-10, jan. 1999.

KOBIERSKA-SZELIGA, M.; CZECZOT, H. Characterization of the genotoxic properties of nitrofurans: nitrofurazone and furazolidone. Acta Biochimica Polonica, v. 41, n.1, p.1-5, 1994.
KOROLKOVAS, A. Dicionário terapêutico Guanabara. Rio de Janeiro: Guanabara Koogan, 2002.

KRAUTH-SIEGEL, R. L.; INHOFF, O. Parasitespecific trypanothione reductase as a drug target molecule. Parasitology Research, v. 90, p. S77S85, 2003. Supplement 2.

KUBINYI, H. QSAR: hansch analysis and related approaches. In: MANNHOLD, R.;

KROSGAARD-LARSEN, P.; TIMMERMAN, H. (Ed.). Methods and principles in medicinal chemistry. Weinheim: VHC, 1993a. v. 1.

KUBINYI, H. (Ed.). 3D QSAR in drug design: theory, methods and applications. Leiden: ESCOM, $1993 b$.

KUBINYI, H. Strategies and recent techonologies in drug discovery. Pharmazie, v. 50, p. 647-662, 1995a.

KUBINYI, H. The quantitative analysis of structureactivity relationships. In: WOLFF, M. E. (Ed.). Burge's medicinal chemistry. 5th ed. New York: Wiley, 1995b. v. 1, p. 497-571.

LIU, J.; PAN, D.; TSENG, Y.; HOPFINGER, A.J. 4D-QSAR analysis of a series of antifungal P450 inhibitors and 3D-pharmacophore comparisons as a function of alignment. Journal Chemistry

Computer Science, v. 43, p. 2170-2179, 2003.

LOCKMAN, J. W.; HAMILTON, A. D. Recent developments in the identification of chemotherapeutics for Chagas Disease. Current Medicinal Chemistry, v. 12, n. 8, p. 945-959, 2005.

MALDONADO, R. A.; KUNIYOSHI, R. K.; LINSS, J. G.; ALMEIDA, I. C. Trypanosoma cruzi oleate desaturase: molecular characterization and 
comparative analysis in other trypanosomatids. Journal of Parasitology, v. 92, v. 5, p. 10641074, 2006.

MCCALLA, D. R. Mutagenicity of nitrofuran derivatives: review. Environmental Mutagenesis, v. 5, n. 5, p. 745-765, 1983.

MECCA, M. M.; DIAZ, E. G.; CASTRO, J. A. Nifurtimox biotransformation to reactive metabolites or nitrite in liver subcellular fractions and model systems. Toxicology Letters, v. 136, n. 1, p. 1-8, 2002.

MEYER, P. Discovering new drugs. In: WERMUTH, C. G. (Ed.). The practice of medicinal chemistry. London: Academic Press, 1996b. p. 11-24.

NARA, T.; KAMEL, Y.; TSUBOUCHI, A. ANNOURA, T.; HIROTA, K.; IZUMI, K.;DOHMOTO, Y.; ONO, T.; AOKI, T. Inhibitory action of marine algae extracts on the Trypanosoma cruzi dihydoorotate activity and on the protozoan growth in mammalian cells. Parasitology

International, v.54, n.1, p. 59-64, 2005.

NWAKA, S.; HUDSON, A. Innovative lead discovery strategies for tropical diseases. Nature

Reviews Drug on Discovery, v. 5, n. 11, p. 941955, Nov. 2006.

OLIVEIRA, R. B.; VAZ, A. B. M.; ALVES, R. O.; LIARTE, D. B.; DONNICI, C. L.; ROMANHA, A. J.; ZANI, C. L. Arylfurans as potencial Trypanosoma cruzi trypanothione reductase inhibitors. Memórias do Instituto Oswaldo Cruz, v. 101, n. 2, p. 169-173, mar. 2006.

OTERO, L.; AGUIRRE, G.; BOIANI, L.; DENICOLA, A.; RIGOL, C.; OLEA-AZAR, C.; MAYA, J. D.; MORELLO, A.; GONZÁLEZ, M.; GAMBINO, D.; CERECETTO, $\mathrm{H}$.
Nitrofurylsemicarbazone rhenium and ruthenium complexes as anti-trypanosomal agents. European Journal of Medicinal Chemistry, v. 41, n. 11, p. 1231-1239, 2006.

OTERO, L.; VIEITES, M.; BOIANI, L.; DENICOLA, A.; RIGOL, C.; OPAZO, L.; OLEA-AZAR, C.; MAYA, J. D.; MORELLO, A.; KRAUTH-SIEGEL, R. L.; PIRO, O. E.; CASTELLANO, E.; GONZÁLEZ, M.; GAMBINO, D.; CERECETTO, H. Novel antitrypanosomal agents based on Palladium Nitrofurylthiosemicarbazone complexes: DNA and redox metabolism as potencial therapeutic targets. Journal of Medicinal Chemistry, v. 49, n. 11, p. 3322-3331, 2006.

PITA, S. S. R.; ALBUQUERQUE, M. G.; ALENCASTRO, R. B.; RODRIGUES, C. R. Modelos de QSAR - 4D de inibidores peptídicos da tripanotiona redutase. In: REUNIÃO ANUALDA SOCIEDADE BRASILEIRA DE QUÍMICA, 29., Águas de Lindóia. [Resumos]. Disponívelem: <https://sec.sbq.org.br/cd29ra/resumos/T12642.pdf>. Acesso em: 25 nov. 2006.

PRATA, A.; DIAS, J. C. P.; COURA, J. R. Os primórdios da doença. Revista da Sociedade Brasileira de Medicina Tropical, v. 44, p. 1-6, 2011. Suplemento2.

PRIETO, J. J.; TALEVI, A.; BRUNO-BLANCH, L. E. Application of linear discriminant analysis in the virtual screening of antichagasic drugs through trypanothione reductase inhibition. Molecular Diversity, v. 10, n. 3, p. 361-375, Aug. 2006.

RAETHER, W.; HÄNEL, H. Nitroheterocyclic drugs with broad spectrum activity. Parasitology Research, v. 90, p. S19-S39, 2003. Supplement 1.

RASSI, A.; LUQUETTI, A. O.; RASSI, G. G.; RASSI JR., A. Tratamento específico da doença de 
Chagas: uma visão de 1962 a 1999. Revista de

Patologia Tropical, v. 29, p.157-163, 2000.

Suplemento.

RAVASCHINO, E. L.; DOCAMPO, R.; RODRIGUEZ, J. B. Design, synthesis, and biological evaluation of Phosphinopeptides against Trypanosoma cruzi targeting Trypanothione Biosynthesis. Journal of Medicinal Chemistry, v.49, n. 1, p. 426-435, 2006.

RIGOL, C.; OLEA-ZAR, C.; MENDIZÁBAL, F.; OTERO, L.; GAMBINO, D.; GONZÁLEZ, M.; CERECETTO, H. Electrochemical and ESR study of 5-nitrofuryl-containing thiosemicarbazones antiprotozoal drugs. Spectrochimica Acta Part A, v. 61, n. 13/14, p. 2933-2938, 2005.

RONDEAU, J. M.; SCHREUDER, H. The use of $\mathrm{X}$-ray structures of receptors and enzymes in drug Discovery. In: WERMUTH, C. G. The practice of medicinal chemistry. London: Academic Press, 1996. p. 485-522.

SANTOS, K. S. C. R.; MATOS, J. R.; FERREIRA, E. I. Synthesis and Chemical Characterization of a Polymeric Produg for Prolonged Release of Nitrofurazone.

Macromolecular Symposia, v. 299-300, n. 1, p. 113-123, 2011.

SCHNARE, M. N.; GRAY, M. W. A candidate U1 small nuclear RNA for trypanosomatid protozoa. Journal of Biological Chemistry, v. 274, n. 34, p. 23691-23694, 1999.

SDR - Strategic Direction for Research, WHO, 2002. Disponível em: http://www.who.int./tdr/ diseases/chagas/directions.htm . Acesso em: 14 fev. 2005.

SERAFIM, E. O. P. Conversão in vitro e in vivo do pró-fármaco hidroximetilnitrofural, candidato a fármaco antichagásico. 2008. 116 f. Tese (Doutorado em Química) - Instituto de Química, Universidade Estadual Paulista, Araraquara, 2008.

SEYDEL, J. K.; SCHAPER, K. J. Chemische struktur und biologishe aktivitat von wirkstoffen: methoden der quantitative strukturwirkung-analyse. Weinheim: VHC, 1979.

SHUKER, S. B.; HAJDUK, P. J.; MEADOWS, R. P.; FESIK, S. W. Discovering high-affinity ligands for proteins: SAR by NMR. Science, v. 274, n. 5292, p. 1531-1534, 1996.

SILVA, A. T. A.; CHUNG, M. C.; CASTRO, L. F.; GÜIDO, R. V. C.; FERREIRA, E. I. Advances in prodrug design. Mini Reviews in Medicinal Chemistry, v. 5, n. 10, p. 893-914, 2005.

SOUZA, V. A.; SILVA, R.; PEREIRA, A. C.; ROYO, V. A.; SARAIVA, J.; MONTANHEIRO, M.; SOUZA, G. H. B.; SILVA FILHO, A. A.; GRANDO, M. D.; DONATE, P. M.; BASTOS, J. K.; ALBUQUERQUE, S.; SILVA, M. L. A. Trypanocidal activity of (-)-cubebin derivatives against free amastigote forms of Trypanosoma cruzi. Bioorganic \& Medicinal Letters, v. 15, n. 2, p. 303-307, 2005.

STOPPANI, A. O. M.; PAULINO, M.; DUBIN, $\mathrm{M}$. Oxigen radicais and the chemotherapy of Chagas'disease: an overview. Ciência e Cultura, v. 48, n. 1/2, p. 75-85, 1996.

SZAJNMAN, S. H.; RAVASCHINO, E. L.; DOCAMPO, R.; RODRIGUEZ, J. B. Synthesis a nd biological evaluation of 1-amino-1, 1bisphosphonates derived from fatty acids agaist Trypanosoma cruzi targeting farnesyl pyrophosphate synthase. Bioorganic \& Medicinal Chemistry Letters, v. 15, n. 21, p. 4685-4690, 2005. 
TDR-TRAINING IN TROPICAL DISEASES. Strategic direction: Chagas Disease, 2005. Disponível em <http://www.who.int/tdr >. Acesso em: 15 jul. 2007.

TESTA, B.; MAYER, J. M. Hidrolysis in drug and produg metabolism, chemistry, biochemistry, and enzymology. Zürich: WiletVCH, 2003. p. 148-162.

TOCHER, J. H. Reductive activation of nitroheterocyclic compounds. General Pharmacology, v. 28, n. 4, p. 485-487, 1997.

TRACY, J. W.; WEBSTER JR., T. Chemoterapy of parasitic infections. In: HARDMAN, J. G.; LIMBIRD, L. E.; GILMAN, A. G. (Ed.). Goodman \& Gilman's the pharmacological basis of therapeutics. 10. ed. New York: McGraw Hill, 2001. p. 1059-1069.

URBINA, J. A.; PAYARES, G.; MOLINA, J.; SANOJA, C.; LIENDO, A.; LAZARDI, K.; PIRAS, M. M.; PIRAS, R.; PEREZ, N.; WINCKER, P.; RYLEY, J. F. Cure of short- and long-term experimental Chagas'disease using DO 870. Science, v. 273, n. 5277, p. 969-971, 2003.

VERLINDE, C. L.; HANNAERT, V.; BLONSKI, C.; WILSON, M.; PÉRIÉ, J. J.; FOTHERGILLGILMORE, L. A.; OPPERDOES, F. R.; GELB, M. H.; HOL, W. G.; MICHELS, P. A. Glycolysis as a target for the design of new anti-trypanosome drugs. Drug Resist Updat, v.4, n. 1, p. 50-65, 2001.
WERMUTH, C. G. The practice of medicinal chemistry. 2. ed. Oxford: Elsevier, 2004.

WILLIAMS-BLANGEROS, S.; VANDEBERG, J. L.; BLANGERO, J.; CORREAA-OLIVEIRA, R.

Genetic epidemiology of Trypanosoma cruzi infection and Chagas disease. Fronteras en Biotecnologia y Bioingeneria, v.8, p. 337-345, 2003.

WOLFF, M. E. (Ed.). Burger's medicinal chemistry and drug discovery: principles and practice. New York: John Wiley \& Sons, 1995. v. 1, $1064 \mathrm{p}$.

WORLD HEALTH ORGANIZATION. Working to overcome the global impact of neglected tropical diseases. Geneva, 2011. p. 1-25.

WORONOWICZ, A.; DE VUSSER, K.; LAROY, W.; CONTRERAS, R.; MEAKIN, S. O.; ROSS, G. M.; SZEWCZUK, M. R. Trypanosome transsialidase targets TrKA tyrosine kinase receptor and induces receptor internalization and activation.

Glycobiology, v. 14, n. 11, p. 987-998, 2004.

ZACKS, M. Impairment of cell division of Trypanosoma cruzi epimastigotes. Memórias do Instituto Oswaldo Cruz, v. 102, n. 1, p. 111-115, feb. 2006.

ZHENG, A.; WANG, W.; ZHANG, H.; WANG, B. Two new improved approaches to the synthesis of coumarin-based prodrugs. Tetrahedron, v. 55, n. 14, p. 4237-4254, 1999.

ReCEBIDO EM 30/12/2010

ACEITO EM 29/4/2011 